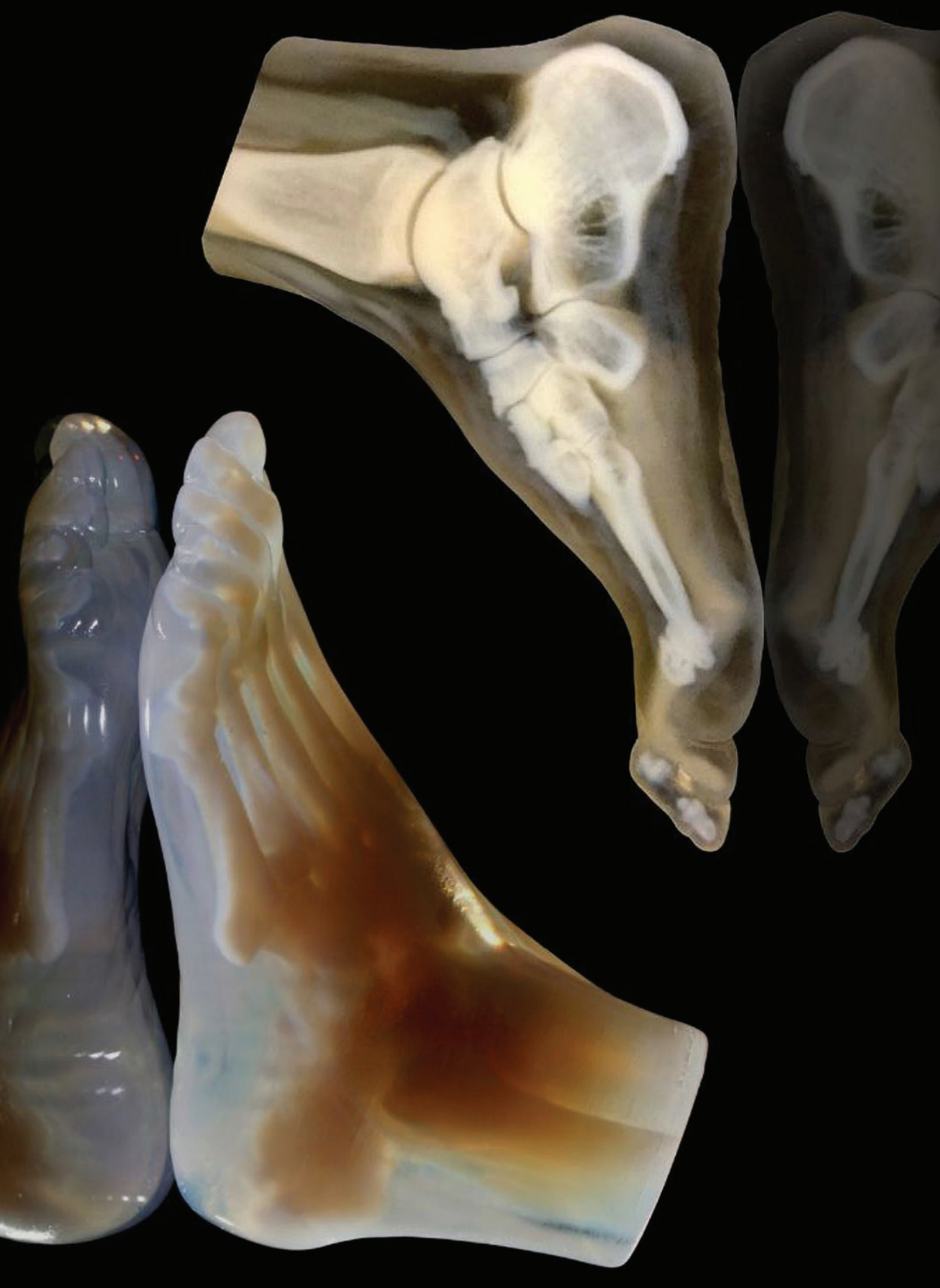




\title{
From Improved Diagnostics to Presurgical Planning: High-Resolution Functionally Graded Multimaterial 3D Printing of Biomedical Tomographic Data Sets
}

\author{
Ahmed Hosny, Steven J. Keating, Joshua D. Dilley, Beth Ripley, Tatiana Kelii, Steve Pieper, ${ }^{6}, 7$ \\ Dominik Kolb,2 Christoph Bader, ${ }^{2}$ Anne-Marie Pobloth, ${ }^{8}$ Molly Griffin, ${ }^{9}$ Reza Nezafat, ${ }^{10}$ \\ Georg Duda, Ennio A. Chiocca, ${ }^{11,12}$ James R. Stone, ${ }^{13}$ James S. Michaelson,14,15 \\ Mason N. Dean, ${ }^{16}$ Neri Oxman, ${ }^{2}$ and James C. Weaver ${ }^{1}$
}

\begin{abstract}
Three-dimensional (3D) printing technologies are increasingly used to convert medical imaging studies into tangible (physical) models of individual patient anatomy, allowing physicians, scientists, and patients an unprecedented level of interaction with medical data. To date, virtually all 3D-printable medical data sets are created using traditional image thresholding, subsequent isosurface extraction, and the generation of .stl surface mesh file formats. These existing methods, however, are highly prone to segmentation artifacts that either overor underexaggerate the features of interest, thus resulting in anatomically inaccurate $3 \mathrm{D}$ prints. In addition, they often omit finer detailed structures and require time- and labor-intensive processes to visually verify their accuracy. To circumvent these problems, we present a bitmap-based multimaterial 3D printing workflow for the rapid and highly accurate generation of physical models directly from volumetric data stacks. This workflow employs a thresholding-free approach that bypasses both isosurface creation and traditional mesh slicing algorithms, hence significantly improving speed and accuracy of model creation. In addition, using preprocessed binary bitmap slices as input to multimaterial 3D printers allows for the physical rendering of functional gradients native to volumetric data sets, such as stiffness and opacity, opening the door for the production of biomechanically accurate models.
\end{abstract}

\footnotetext{
${ }^{1}$ Wyss Institute for Biologically Inspired Engineering, Harvard University, Cambridge, Massachusetts.

${ }^{2}$ MIT Media Lab, Cambridge, Massachusetts.

${ }^{3}$ Department of Anesthesia, Critical Care and Pain Medicine, Harvard Medical School, Massachusetts General Hospital, Boston, Massachusetts.

${ }^{4}$ Department of Radiology, University of Washington, Seattle, Washington.

${ }^{5}$ Department of Radiology, University of California, San Francisco, California.

${ }^{6}$ Surgical Planning Laboratory, Department of Radiology, Brigham and Women's Hospital, Boston, Massachusetts.

${ }^{7}$ Isomics, Inc., Cambridge, Massachusetts.

${ }^{8}$ Department of Biomechanics and Musculoskeletal Regeneration, Julius Wolff Institute and Berlin-Brandenburg Center for Regenerative Therapies, Charité-Universitätsmedizin Berlin, Berlin, Germany.

${ }^{9}$ Division of Surgical Oncology, Gillette Center for Women's Cancers, Massachusetts General Hospital, Boston, Massachusetts.

${ }^{10}$ Department of Medicine (Cardiovascular Division), Beth Israel Deaconess Medical Center, Harvard Medical School, Boston, Massachusetts.

${ }^{11}$ Department of Neurosurgery at Harvard Medical School and ${ }^{12}$ Department of Neurosurgery and Institute for the Neurosciences at Brigham and Women's Hospital, Center for Neuro-oncology at Dana-Farber Cancer Institute, Boston, Massachusetts.

${ }^{13}$ Department of Pathology, Massachusetts General Hospital, Harvard Medical School, Boston, Massachusetts.

${ }_{15}^{14}$ Department of Pathology and Surgery, Massachusetts General Hospital, Boston, Massachusetts.

${ }^{15}$ Department of Pathology, Harvard Medical School, Cambridge, Massachusetts.

${ }^{16}$ Department of Biomaterials, Max Planck Institute of Colloids and Interfaces, Potsdam, Germany.
}

Opposite page: Using bitmap-based 3D printing of tomographic medical data sets, it is possible to obtain an unprecedented level of detail in patient-specific anatomical models. One such example, a 3D-printed foot (lower), and its cross-section (upper), clearly reveal the intricate architecture of the different bone types, as well as the structural details of the surrounding soft tissues. Photo Credit: Steven Keating and Ahmed Hosny/Wyss Institute at Harvard University. 
Keywords: 3D printing, bitmap printing, biomedical imaging, tomography, CT, MRI

\section{Introduction}

FROM THE INCEPTION of X-ray imaging in the late $1890 \mathrm{~s}$ through more recent advances in high-resolution CT and MRI scanning, medical imaging has been driven by a continued need for high-quality accurate representations of anatomical structures. In recent years, there has been increased interest in transcending the two dimensional environments of radiographic prints and computer screens through translation of digital images into tangible (physical) objects through threedimensional (3D) printing. ${ }^{1}$

3D-printed models have been used for presurgical planning in almost all surgical subspecialties, ${ }^{2-9}$ allowing for the precise planning and simulation of surgical approach, incision, and hardware sizing and placement-thereby reducing the need to perform these steps intraoperatively. ${ }^{10-12}$ Physical models can also serve as cutting guides for resection and as templates for shaping of reconstruction hardware, implants, and prostheses to fit a patient's anatomy. ${ }^{13-15}$ In addition, 3D printing has also been used to produce detailed models that offer realistic anatomy and capture patient variability for education and training, ${ }^{16}$ and provide easily interpretable visual guides for improving doctor-patient communication. ${ }^{17}$

Despite the clinical utility of 3D-printed models, however, there are several technical challenges that prevent their widespread adoption, particularly with regard to the methods by which the structures to be printed are specified. Typically, features of interest in source biomedical data sets (e.g., crosssectional raster-based biomedical images or volumes from MRI or CT DICOM files) are first isolated from the surrounding tissues, either through a time-consuming manual segmentation approach or through the use of specific intensity thresholds that best capture the feature(s) of interest. ${ }^{18}$ The result of this filtering step is used to generate a single isosurface that is then translated into a printable format [i.e. stereolithography (.stl) file, the current standard for 3D printing workflows], which describes surface geometries through mesh representations of face vertices and normals.

This reduction of complex anatomy to a single surface is necessary due to a constraint of traditional 3D printers, which can often only output a single material. As a result, printing is a presence-absence task: data set pixels contained within the isosurface are printed and those beyond the isosurface boundary are excluded. This simplification, unfortunately, ignores local pixel-level intensity differences native to most medical imaging data, such as those resulting from subtle gradients in tissue properties. Furthermore, in order for thresholding to be successful, the target structures must be relatively homogeneous and simultaneously distinct from adjacent structures, which is seldom the case. As a result, isosurfaces generated from thresholding often exhibit non-natural discontinuities (e.g., in regions of lower mineral density) and/or apparent tissue links to adjacent structures. In addition, fine structures at the limits of imaging resolution are particularly susceptible to errors introduced from thresholding, which can cause portions of the structure to appear to be missing when actually present.
Consequently, even the smallest gap in a fine structure can lead to significant error or nonprintability of an .stl file, requiring the user to digitally generate regions of the structure that are often not present in the original data. As a result, this approach can frequently lead to anatomically inaccurate 3D representations.

To help mitigate some of these issues, and by allowing the mixing of two or more different printing materials in numerous predefined ratios (e.g., in inkjet-based 3D printers), or through the use of numerous distinct source materials (e.g., in filament-based 3D printers), multimaterial 3D printers can produce models that incorporate multiple .stl files. This approach greatly expands the number of different anatomical structures that can be represented by different colors or materials (e.g., through the use of multiple thresholds) and, as such, can improve the interpretive value of 3D-printed anatomical models. However, the printing of multiple nested .stl files is labor intensive, computationally expensive, and ultimately nonscalable. To correctly represent routine medical CT data, for example, 4096 different material mixtures (corresponding to the 12-bit discretization of Hounsfield unit radiodensity values typically recorded) would need to be defined and associated with 4096 separate .stl files.

To remedy these limitations, recent efforts have explored multiple bitmap processing and dithering techniques for $3 \mathrm{D}$ printing as a means of bypassing isosurface generation and slicing algorithms. Such methods have found applications in the printing of data-driven heterogeneous materials, ${ }^{19}$ fullcolor prints on multijet ${ }^{20}$ and fused deposition modeling printers, ${ }^{21}$ and the design and fabrication of prosthetic sockets with locally varying material stiffness. ${ }^{22}$ In this study, we demonstrate the utility of bitmap-based workflows in the 3D printing of biomedical data sets. The proposed workflow allows for the rapid and highly accurate generation of physical models from cross-sectional image stacks, while utilizing a thresholding-free approach. By using preprocessed bitmap slices as direct 3D printer inputs, fine details and biomechanical gradients such as mineral density are preserved, with the added benefit of a significant reduction in the time needed for the generation of printable data files.

\section{Materials and Methods}

\section{Data acquisition and processing}

This study was IRB approved, and since all CT and MRI data sets from either living patients or from excised tissue samples were anonymized before image processing, the requirement for individual informed consent was waived.

A summary of the bitmap-based data processing workflow is outlined in Figure 1 and compared with the traditionally implemented .stl-based approach. The methodology is based on establishing format compatibility between the data outputs from biomedical imaging processes and the inputs required for multimaterial UV-cured liquid photopolymer 3D printing processes. As a result, operations employed in traditional 3D printing workflows that can result in data loss are bypassed. 


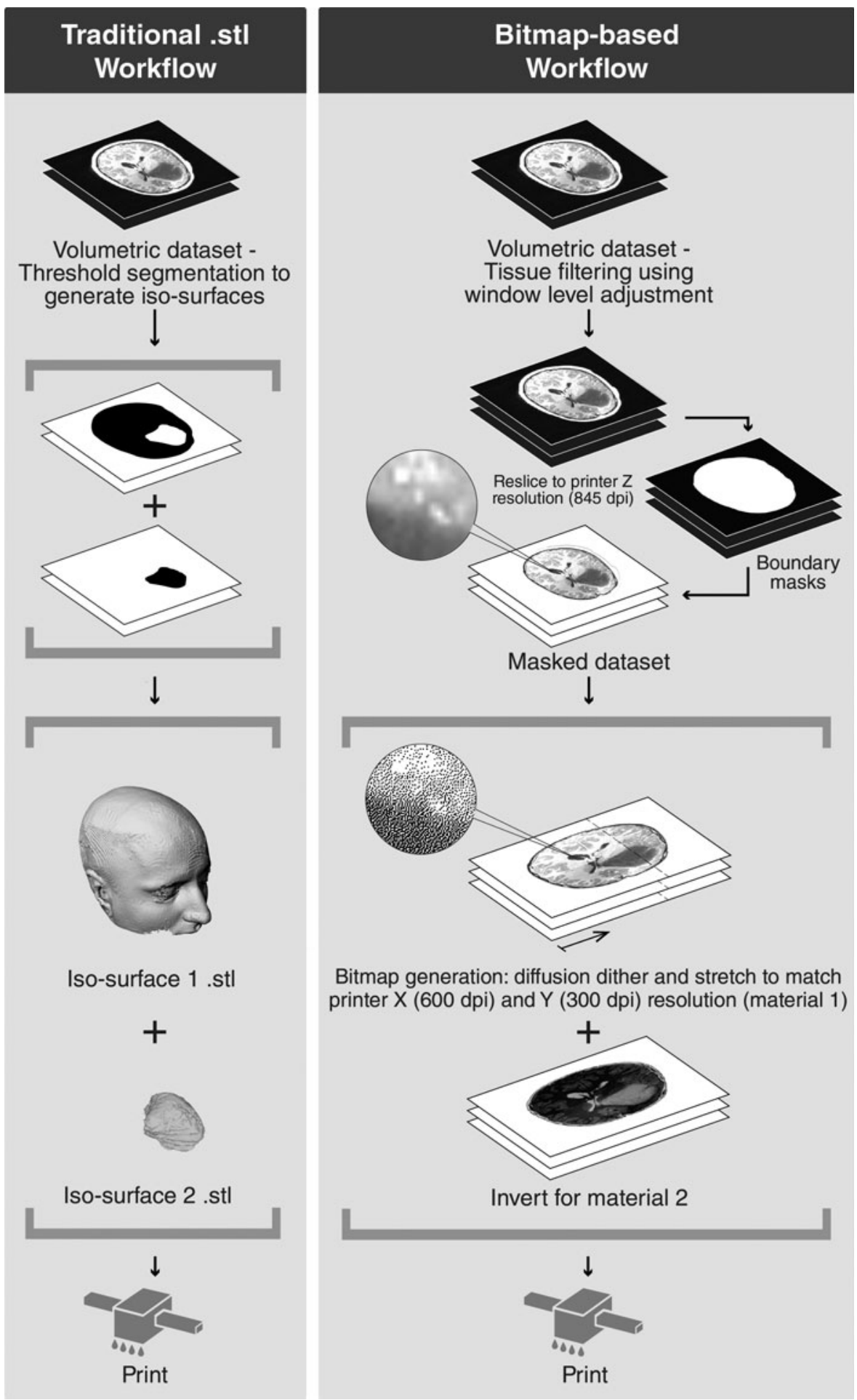

FIG. 1. Traditional .stl generation workflow (left) versus bitmap-based workflow (right). In contrast to traditional isosurface generation-based workflows (left), the bitmap-based 3D-printing approach (right) faithfully preserves the subtle grayscale intensity gradients from native tomographic image stacks. 3D, three-dimensional; .stl, stereolithography.

Medical imaging DICOM files were first processed with the open-source 3DSlicer software package, ${ }^{23}$ where window/level adjustments were used to emphasize/de-emphasize specific features or areas of interest: a wider window width captures a larger range of displayed values, whereas a nar- row window can be used to highlight soft tissue details. After the desired window/level adjustments for a specific data set of interest were performed by a trained medical professional, the images were further processed as numerical arrays in Python. Since slice thickness in biomedical 

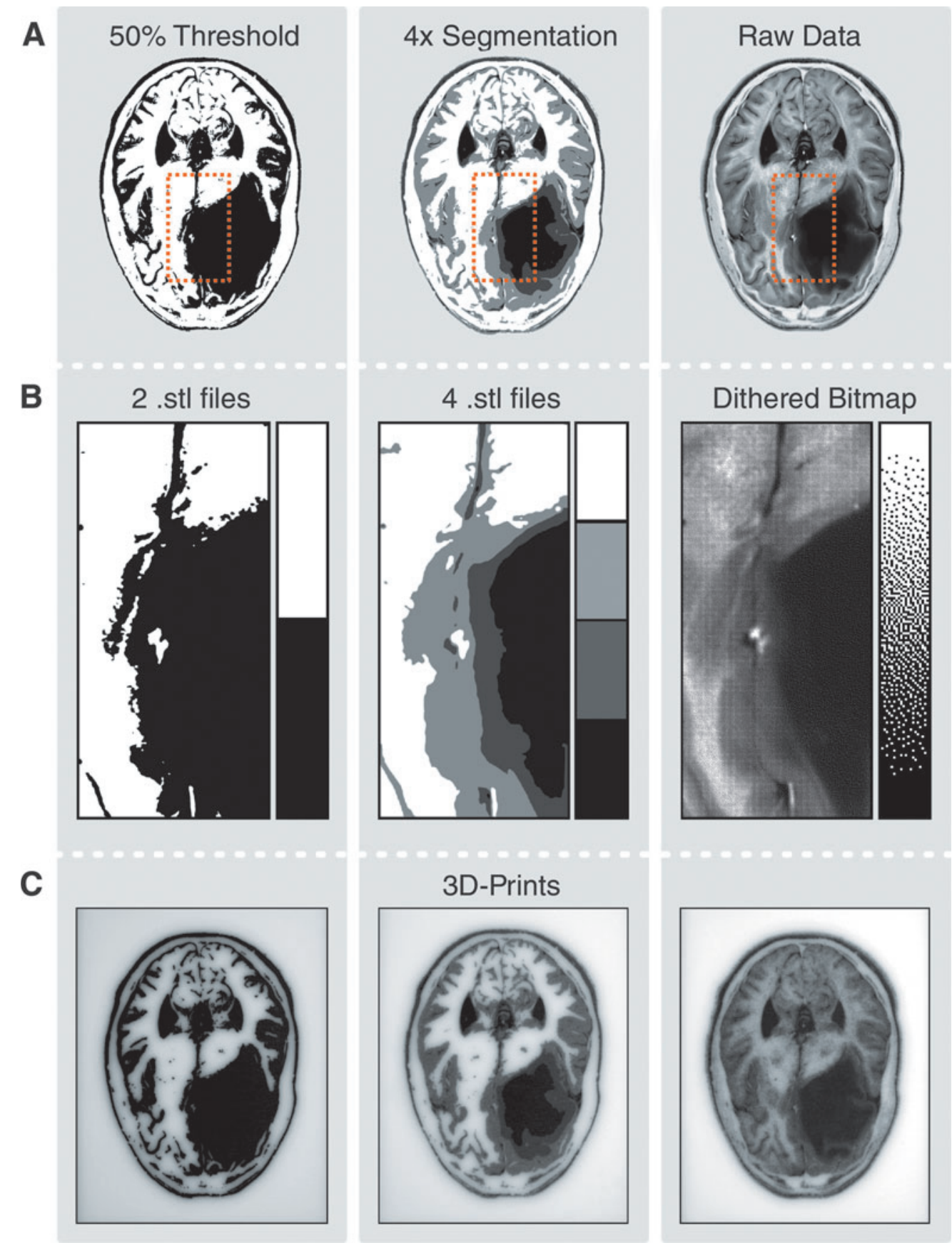

FIG. 2. Three different 3D-printing approaches for the preparation of MRI data taken from a patient with an astrocytoma brain tumor (viewed in a top-down manner). The same transverse (axial) slice from the raw MRI data (cf. Fig. 3) is used for comparison of the three printing approaches (arranged in columns), as they progress from the source data [(A) slice overview, and (B) the orange boxed regions shown at higher magnification], to $(\mathbf{C})$ the final 3D-printed result. The first method (left) uses a single threshold to convert a grayscale domain into a binary domain, which is equivalent to the traditional sstl file generation protocol. Notice the artifacts that emerge as a result of the oversimplification of the original data set, including an exaggeration of the tumor size. Four thresholds are created in the second (middle) method, equivalent to printing using multiple .stl files. Although this clearly represents a wider range of grayscale information, artifacts are not entirely eliminated and new structural boundaries not present in the original data are introduced. The third method (right) employs diffusion dithering on the original MRI data without any thresholding, and thus maintains the fine-scale gradients present in the source data. 
A High-throughput Tissue Filtering

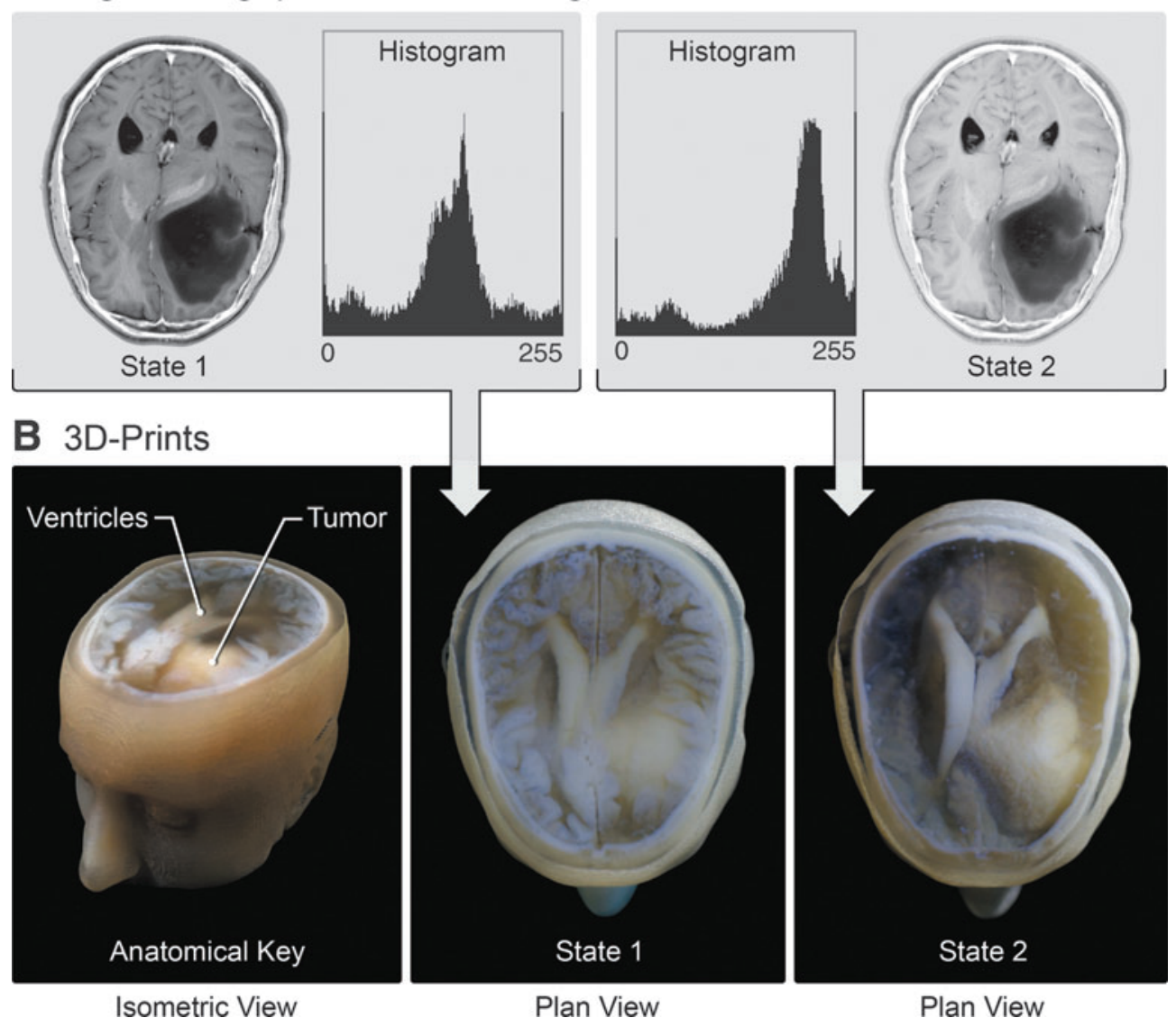

FIG. 3. High-throughput tissue filtering in bitmap-based data sets. By adjusting image histograms (window level adjustments) before dithering into binary bitmaps, it is possible to control the visibility and/or transparency of specific features of interest within a resulting 3D-printed model. (A) Single (axial) MRI slice and its accompanying histogram (left), and its counterpart after the application of a window level adjustment (right). (B) In the resulting 3D-printed model based on the original, unfiltered slice data (printed using a combination of transparent and white materials), the tumor is mostly obscured by the surrounding brain parenchyma. In contrast, 3D printing of the window level-shifted data set (right) increases the optical transparency of the surrounding brain parenchyma to reveal the structural details of the underlying tumor and its physical distortion of the adjacent ventricles. An isometric view (left) of the 3D-printed model is provided to better illustrate the details of the external facial geometry and provide an anatomical key to the prominent features of interest.

imaging data sets can vary widely (usually ranging from 1 to $5 \mathrm{~mm}$ ), the data sets were resliced at a $30-\mu \mathrm{m}$ interval to match the $30-\mu \mathrm{m}$ slice thickness of the multimaterial $3 \mathrm{D}$ printer used in this study. This process was achieved using the SimpleITK library's linear interpolation function to fill in missing slices.

To delineate the outer contours of the 3D-printed objects, a threshold was used to discard values representing air (e.g., in cases where the skin or other exterior surface provided a natural data boundary), resulting in a stack of masks that was simply subtracted from the imaging data (Fig. 1). Alternatively, the inherent boundaries of the data set could be used directly (e.g., rectilinear for most CT and MRI and conical for 3D ultrasound). The masked data sets were then scaled into the printer's native $\mathrm{X}$ and $\mathrm{Y}$ resolutions of 600 and $300 \mathrm{dpi}$, respectively, by stretching the data along the $\mathrm{X}$ direction. Finally, the images were converted into binary bitmaps of black [1] and white [0] pixels using the Floyd-Steinberg dithering algorithm. ${ }^{24}$ This process results in the integrated density ratio of black to white pixels in the new images, approximating the grayscale values in the original source files, conceptually similar to the use of halftone image processing techniques in newsprint. When 3D printing with two materials (e.g., black and white or opaque and transparent photopolymers), two bitmap stacks must be created-one for each materialone stack being the inverse of the other. For the purposes of comparison, a series of data sets were also segmented using traditional threshold-based segmentation and converted to .stl files (also using 3D Slicer) for accurate benchmarking.

\section{$3 D$ printing}

All models were produced using a commercially available Connex500 multimaterial 3D printer (Stratasys, Rehovot, Israel). Depending on the desired properties and color range 

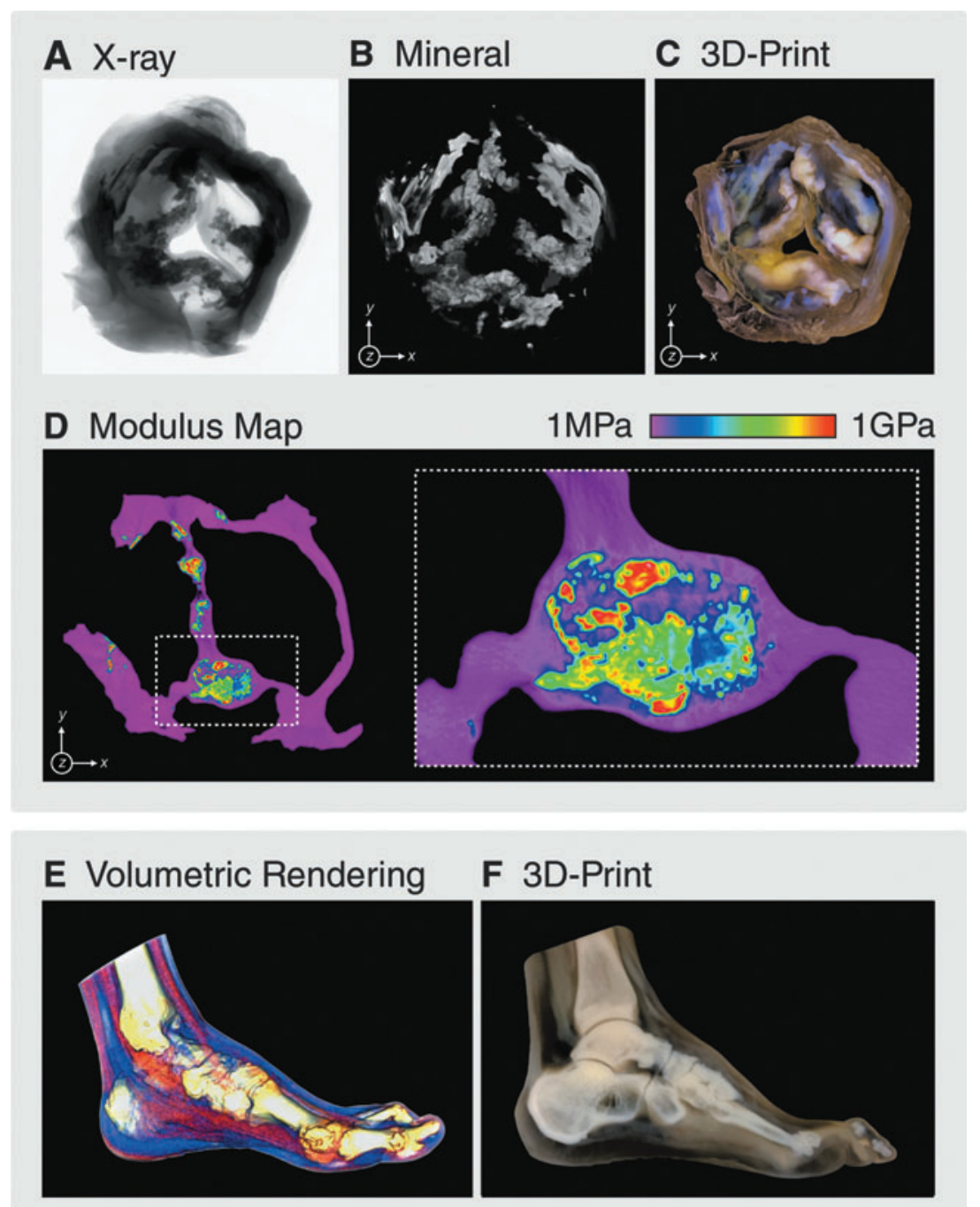

\section{F 3D-Print}

G Modulus Map
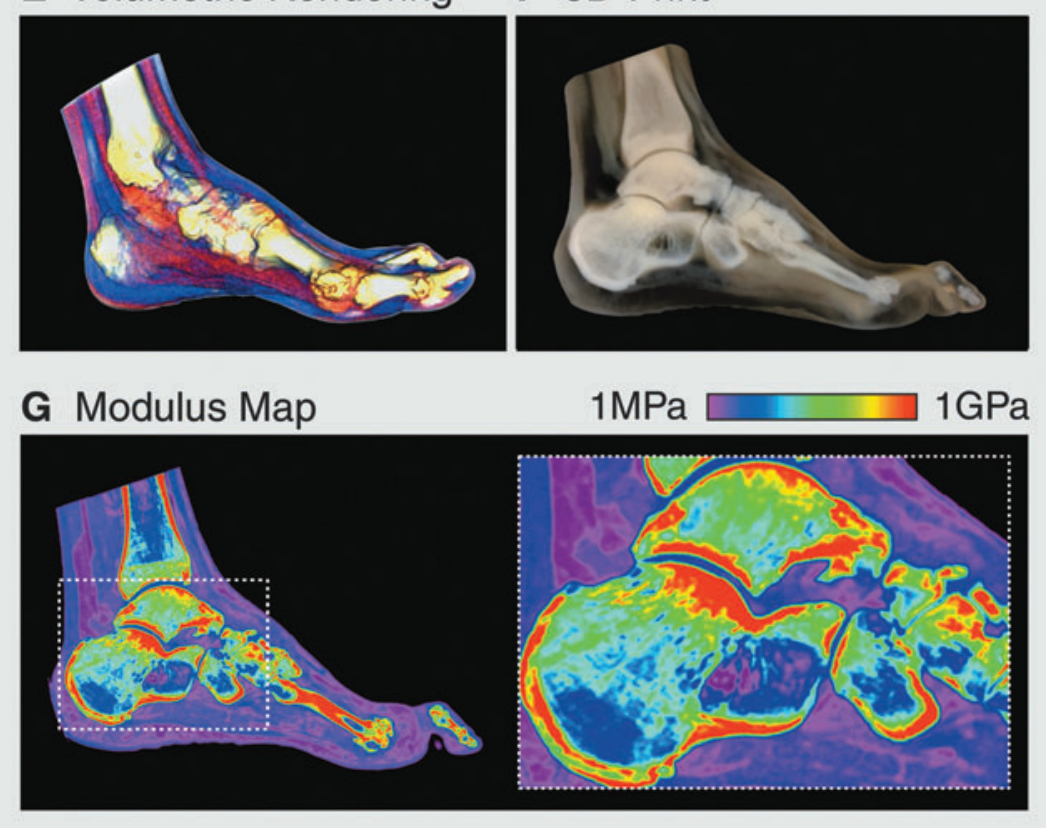

FIG. 4. Variable modulus multimaterial 3D printing through the digital mixing of rigid and flexible photopolymers. (A) Radiograph of an ex vivo aortic valve with senile calcification, (B) its digitally isolated mineral inclusions, and (C) a corresponding 3D-printed model clearly illustrating the distribution, mineral density, and ultrastructure of the calcified plaques. (D) Modulus maps of a single (sagittal) slice through the aortic valve [taken midway down the $\mathrm{z}$-axis as referenced in (C)], wherein the lowest Hounsfield units are assigned to a low modulus (flexible) material and the highest Hounsfield units are assigned a high-modulus (rigid) material. In contrast to traditional segmentation approaches, the subtle differences in calcification density are faithfully preserved. (E) Volumetric rendering of a human foot, highlighting the tendons and ligaments, which would be challenging to segment using a traditional stl file-based workflow. (F) Employing the bitmapbased approach, the resulting sectioned 3D-printed model clearly reveals the fine-scale architecture of the trabecular and cortical bone as well as the surrounding soft tissue. (G) The accompanying single slice modulus maps reveal the subtle mechanical gradients present in the resulting 3D-printed model. 
A 3D-Rendering

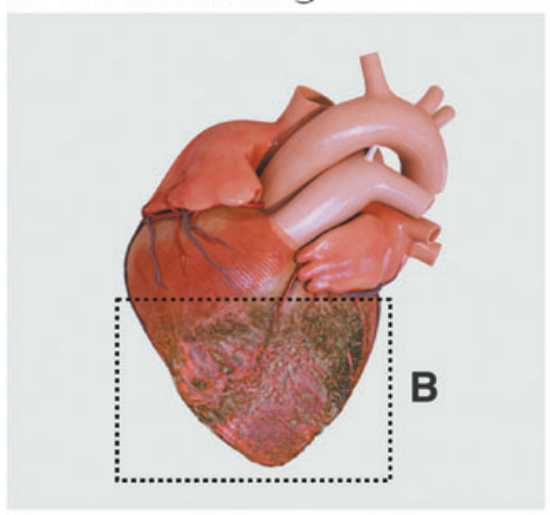

C Vascular Detail

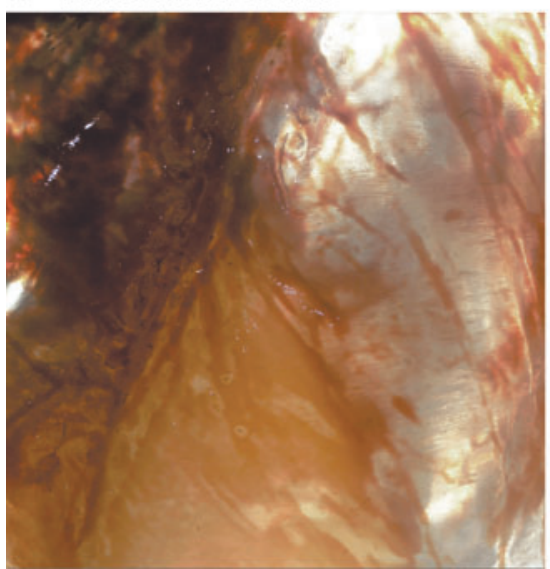

B 3D-Print

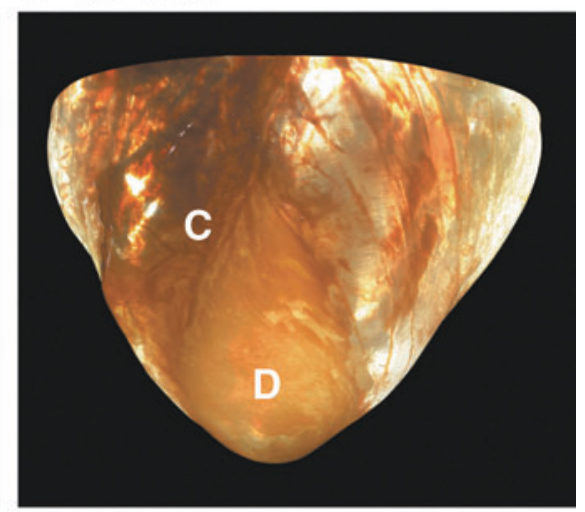

D Scar Tissue Detail

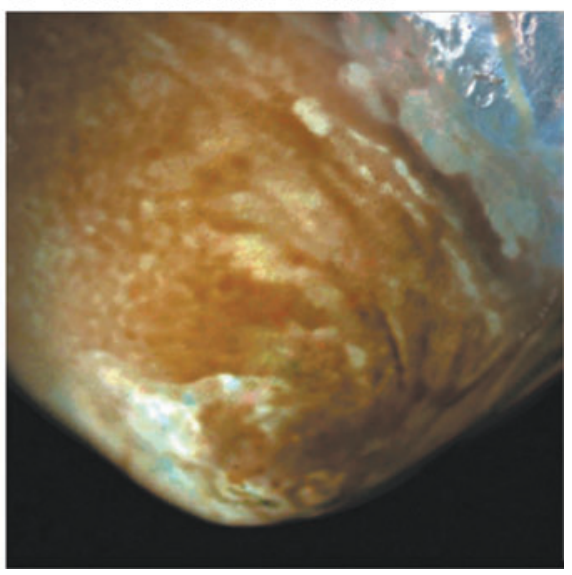

FIG. 5. High-spatial resolution 3D-printed structural details in a pathological heart model. (A) Volumetric rendering of an infarcted heart and (B) the corresponding (cropped) 3D print of its apex, clearly showing (C) the structural organization of the superficial coronary vasculature and (D) the details of the left ventricular scar.

of the printed objects, either high-modulus (RGD835, RGD875, and RGD810) or low-modulus (FLX930 and FLX980) photopolymers of specific colors and transparencies were employed as the constituent materials. Although each material is being deposited on a binary matrix that is the inverse of the other, due to the high-spatial resolution of the printer and microscale (intervoxel) mixing occurring during printing, gradients with smooth transitions are achieved.

\section{Results}

As a test case, we compared the performance of the bitmap-based approach against existing segmentationbased 3D printing workflows in the creation of a patientspecific 3D-printed model of a single MRI slice from a volumetric data set (Fig. 2). The model, a patient with a brain tumor (astrocytoma) in the left frontal lobe, was created using three strategies: (1) traditional 50\% thresholding and isosurfacing, (2) creation of four separately segmented .stl files that corresponded to four distinct grayscale intensity ranges, and (3) the bitmap printing-based approach described here.

Traditional 50\% thresholding and isosurfacing introduced significant imaging artifacts. For example, the size of the tumor was overexaggerated and some distinct brain features with dissimilar grayscale intensity values were depicted as exhibiting similar contrast in the printed model. These errors were partially remedied by using four .stl files to represent four distinct grayscale intensity ranges. However, this approach still resulted in the creation of artificial boundaries that were not present in the native data. In contrast, for the bitmap-based approach, where the dithered bitmap was printed at the printer's native resolution, all of the perceived grayscale intensity gradients were faithfully preserved due to localized material mixing at the microscale.

This bitmap-based 3D printing approach is generally applicable to a wide variety of grayscale data types, and can be further tailored through the use of image filters/corrections and printing media that enhance or de-emphasize target tissues. For example, to improve visualization of deeply embedded structures within a model, the optical transparency of tissue types with specific grayscale intensity ranges can be globally adjusted by simply applying a window level adjustment to the data (Fig. 3). To illustrate this point for the same brain tumor data set already described, the resulting models were printed using a combination of transparent and opaque resins. Instead of deleting 


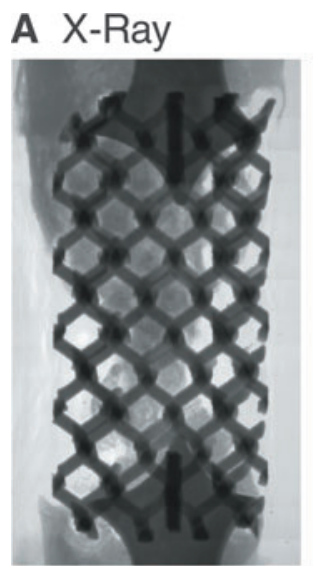

\section{B SEM}

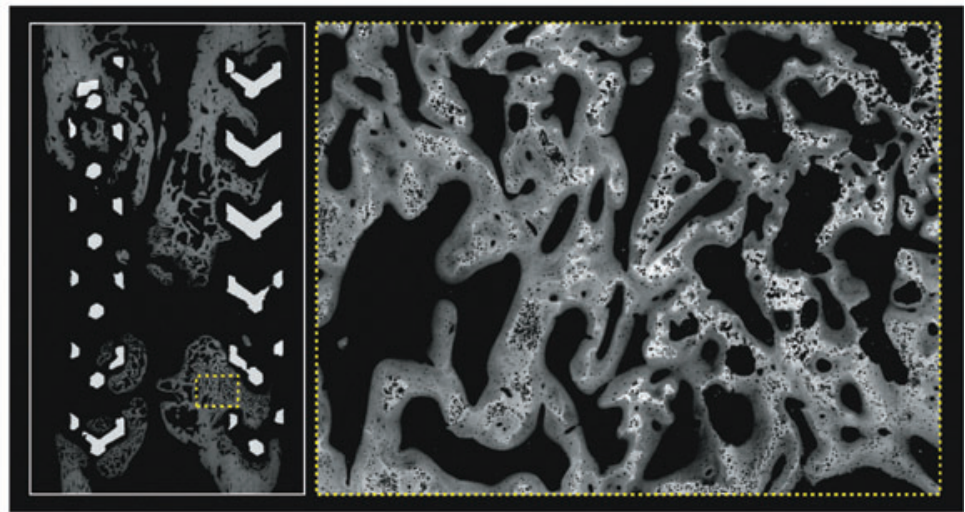

C 3D-Print

D Modulus Map

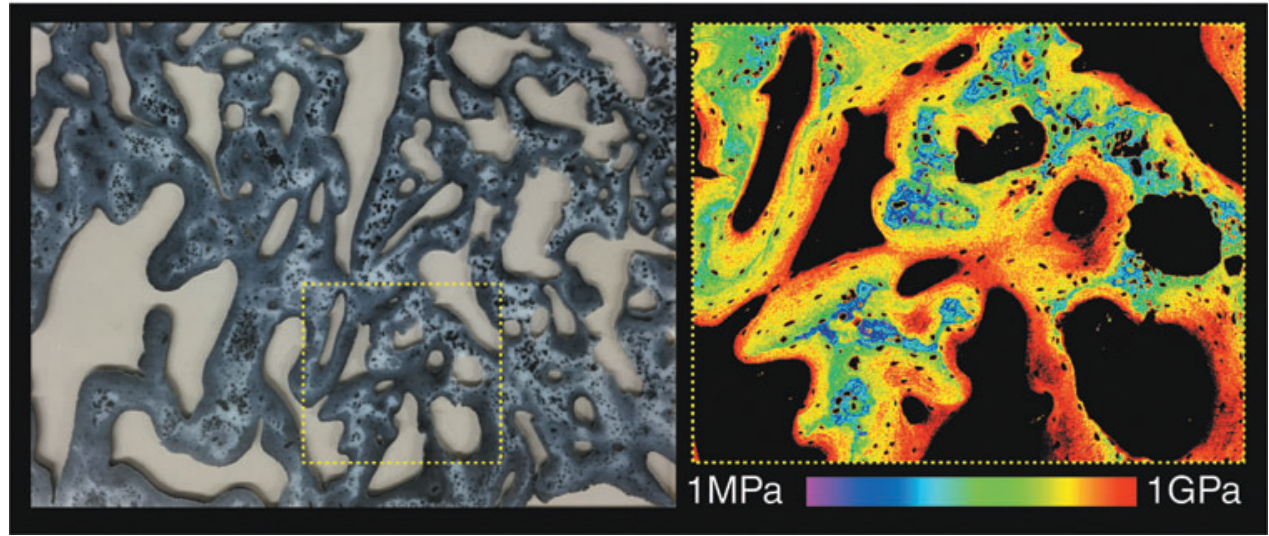

FIG. 6. 3D printing of scanning electron microscopy (SEM)-based data sets. (A) X-ray transmission image and (B) a corresponding backscattered SEM slice of a healing critical defect in an ovine femur immobilized with a titanium cage implant together with a higher magnification single slice backscattered electron micrograph. (C) A 3D-printed counterpart of the SEM image and (D) the corresponding higher magnification modulus map from the resulting 3D-printed model reveal the subtle differences in mineral density during the early stages of bone healing.

a specific range of intensities (as is typically the case during image thresholding), the grayscale intensity values of the gray and white brain matter obstructing the features of interest were only partially reduced to reveal the underlying tumor (depicted with white material) in the 3D-printed model (Fig. 3). In this example, the total time required to perform this correction was on the order of a few minutes, significantly streamlined relative to traditional model generation workflows (Fig. 1).

Although these first two examples of bitmap-based 3D printing focused on MRI data, we next investigated whether this technique could at least partially replicate the variable mechanical properties of tissues that are often represented by local differences in material electron density within X-raybased imaging approaches such as CT (Fig. 4). We have shown in a previous study ${ }^{25}$ investigating the printability of functionally graded engineering composites through multimaterial 3D printing that local variation in a final print's modulus can be achieved by controlling the mixing ratios of high- and low-photoinitiator-containing prepolymers (corresponding to hard and soft materials, respectively) at the microscale. From these known mixing ratios, it is then pos- sible to back calculate the grayscale intensity value from the dithered black:white pixel ratios that would produce such properties. This information can then be used to map out the resulting mechanical properties for each grayscale slice in a CT data set. It is important to note, however, that the applications described in the following examples using biomedical data sets are only intended to approximate the relative, rather than the absolute, tissue-level mechanical properties in the resulting 3D-printed objects. Despite these limitations, we tested the feasibility of this approach by applying the bitmapbased workflow to the $3 \mathrm{D}$ printing of a tricuspid aortic valve with pathological mineralization (Fig. 4A-D) to create a valve model that exhibited mechanical properties that approximated the subtle calcification gradients present in the native sample. These subtle variations in mechanical property of the 3D-printed model (which reflected local variability in the degree of mineralization) would not be achievable using standard .stl generation approaches, as a separate file would need to be created for each modulus range, eliminating mechanical gradients present in the native data.

Figure 4E-G illustrates a similar approach for an adult foot, in this case from a healthy individual. The resulting 
3D-printed model and its corresponding single slice modulus maps capture all of the native resolution of the CT source files, including the detailed trabecular architecture of the bones and the accompanying muscles and tendons. For comparison, a workflow that implemented an .stl file-based approach for the segmentation of all of the cortical and trabecular bone, the marrow, the muscles, tendons, soft tissue, and skin, required $>30 \mathrm{~h}$ and a massive amount of manual segmentation to complete, whereas the bitmap-based approach described here required $<1 \mathrm{~h}$ to perform.

The bitmap-based approach can also be particularly useful for revealing fine-scale structural details in biomedical data sets. For example, the bisected heart model shown in Figure 5 was generated from an ex vivo MRI data set of a swine model of myocardial infarction. The resulting 3D model clearly illustrates the complex 3D geometry of the scar tissue as well as the details of the adjacent coronary vasculature. These types of fine structures are exceedingly difficult to print with meshed files, which require an uninterrupted isosurface. To render these same structures printable with traditional meshdependent workflows, a user would have to edit the mesh to create surface continuities that were not actually present in the native data.

This bitmap-based approach can also be used on nontraditional 3D data sets, such as the backscattered electron micrographs (Fig. 6A-E) of a milled cross section through a healing ovine long bone critical defect surrounded by a titanium cage implant. ${ }^{26}$ In backscattered electron micrographs, as is the case for X-ray-based CT data, contrast varies as a function of electron density, which in the bone case presented here, corresponds to variability in the extent of mineralization. Using these small-scale digital data sets, it is possible to generate large-scale 3D-printed tangible representations of microscale variability in mechanical properties, which could, in turn, be subjected to direct mechanical testing to qualitatively investigate load-bearing capacity and potential failure modes at the bone-implant interface during the early stages of bone healing and growth.

\section{Discussion}

Despite the improved spatial understanding of complex medical data sets afforded by 3D printing, its overall adoption rate has been limited, in large part, by the challenges associated with the translation of medical imaging studies into printable files. Image segmentation can be time consuming and often requires a subjective assessment of where structures of interest truly start and end, especially with subtle gradations or noise in the source data. Furthermore, the process of segmentation can irreversibly remove a portion of the data, which can impact the accuracy of the final 3D-printed model, the extent of which can vary widely as a function of the segmentation strategy employed and/or the skills of the operator performing the segmentation.

The bitmap-based workflow described here, in contrast, does not require a data segmentation step, and thus provides an efficient work-around through the combined integration of data dithering techniques and multimaterial 3D printing. Bitmap-based 3D printing also allows for the enhanced physical visualization of complex and biologically accurate functional gradients, and thus opens the door for the application of this technology to new fields of medical research. For example, the myocardial infarction model depicted in Figure 5 can aid in the visualization of scar geometry, which is normally poorly represented in 3D-printed models. During presurgical planning for ablation-based therapies, this approach could thus potentially improve the ability to identify the viable (conductive) channel in the scar tissue, which can be responsible for ventricular arrhythmia and sudden cardiac death. In addition, the ability to replicate functional mineralization gradients in diseased vessels or heart valves (e.g., in Fig. 4) may permit the direct presurgical evaluation of physical interactions between native valvular mineralization and replacement valve hardware.

The bitmap-based workflow also provides a highly adaptable method for approaching the $3 \mathrm{D}$ visualization of complex properties in medical data sets, through the development of custom resin formulations. For example, the production of more robust 3D-printable photopolymers could potentially lead to the biomechanical mimicking of the elasticity of tendons and ligaments in 3D-printed models, allowing for stress and strain testing and repair planning. In addition to the opacity and elasticity gradients described here, models in the future may also incorporate gradients in density, conductivity, permeability, biodegradability, and other material properties. These new fabrication capabilities could also facilitate the development of personalized active devices, including embedded electronics acting as sensors or actuators, or custom micro- and millifluidics 27,28 with dynamic feedback and patient-specific drug delivery strategies.

It is important to note that the methods for the preparation of the bitmap input files described here were all performed with open-source software using existing image-processing algorithms, thus allowing for the unconstrained widespread adoption of this approach. The ability to openly modify these workflows also permits further experimentation, as different window/level adjustments and dithering algorithms could be explored as a means of producing fully customizable and application-specific 3D prints.

Despite the advantages of this bitmap-based workflow compared with its traditional .stl-based counterpart, there are still some limitations to the technique. For example, although our 3D-printed models can faithfully replicate the details of their native CT or MRI source files, their ability to successfully depict anatomical features of interest is directly limited by the inherent resolution of each imaging modality. Inaccuracies can also be introduced by image reconstruction methods designed to optimize human image viewing rather than 3D printing, and traditional clinical tomography file compression and archival protocols will, in turn, need to be modified as biomedical 3D printing becomes more mainstream. Data set-specific issues also arise with regard to signal intensity and range. For instance, although Hounsfield values in CT are predefined in terms of range, MRI intensities are highly dependent on tissue- and operator-specific parameters. This variability hinders efforts aiming at accurately mapping contrast/intensity achieved on a workstation monitor to those of the final 3D print, and often results in lengthy trial-and-error approaches and lookup tables that do not generalize well across data sets and medical institutions. Recent advances in point cloud-based volumetric data 
processing workflows ${ }^{29}$ however, could help remedy these issues by providing better digital simulation of the appearance and behavior of tangible models of patient-specific anatomy before $3 \mathrm{D}$ printing.

The workflow described here solves problems that constrain traditional biomedical 3D-printing approaches, including the introduction of thresholding artifacts, time costs with segmentation, computationally limited file sizes, the artificial filtering or obscuring of data, and the inability to print small-scale variations in optical transparency and mechanical gradients. Although the models used in this study successfully mimicked grayscale gradients present in native tomography data, recent advances in high-spatial resolution CT and MRI scanning, photorealistic volumetric rendering algorithms, ${ }^{30}$ and full-color bitmap-based printing approaches will allow much more life-like 3D-printed depictions of patient-specific anatomy, and is an area of active research.

By lowering barriers to the visualization of fine details in biorealistic 3D-printed models, we hope to broaden access to this technology for a wide range of medical professionals and patients. When combined with high-resolution biological imaging data, multimaterial medical $3 \mathrm{D}$ printing has the potential to improve treatment, enhance communication, and open new research avenues in precision medicine.

\section{Acknowledgments}

This work was supported by a grant from the Human Frontier Science Program (HFSP) to M.N.D. and J.C.W. under award No. HFSP RGY0067, National Heart, Lung, and Blood Institute grants 1R21HL127650-01 and 1R01HL129185-01 to R.N., and National Institute of Biomedical Imaging and Bioengineering (NIBIB) grant P41 EB015902 to S.P. M.N.D. was supported by DFG-FR 2190/4-1 Gottfried Wilhelm Leibniz-Preis 2010. This work made use of the Connex500's bitmap printing capabilities through a joint collaboration with Stratasys. We thank Brigham and Women's Hospital, Massachusetts General Hospital, and the patient participants for providing the imaging data used in these studies.

\section{Author Disclosure Statement}

C.B., D.K., J.C.W, and N.O. are authors on a patent application filed by the Massachusetts Institute of Technology that describes methods similar to those presented here (application no. 15/628,635; publication no. 20170368755; filed 20 June 2017; published 28 December 2017). All other authors declare that they have no competing interests.

\section{References}

1. Marro A, Bandukwala T, Mak W. Three-dimensional printing and medical imaging: A review of the methods and applications. Curr Probl Diagn Radiol 2016;45:2-9.

2. Cui J, Chen L, Guan X, et al. Surgical planning, threedimensional model surgery and preshaped implants in treatment of bilateral craniomaxillofacial post-traumatic deformities. J Oral Maxillofac Surg 2014;72:1138.e1-e14.

3. Hurson C, Tansey A, O'Donnchadha B, et al. Rapid prototyping in the assessment, classification and preoperative planning of acetabular fractures. Injury 2007;38:1158-1162.
4. Jacobs S, Grunert R, Mohr FW, Falk V. 3D-Imaging of cardiac structures using 3D heart models for planning in heart surgery: A preliminary study. Interact Cardiovasc Thorac Surg 2008;7:6-9.

5. Kono K, Shintani A, Okada H, Terada T. Preoperative simulations of endovascular treatment for a cerebral aneurysm using a patient-specific vascular silicone model. Neurol Med Chir 2013;53:347-351.

6. Mottl-Link S, Hübler M, Kühne T, et al. Physical models aiding in complex congenital heart surgery. Ann Thorac Surg 2008;86:273-277.

7. Eltorai AEM, Nguyen E, Daniels AH. Three-dimensional printing in orthopedic surgery. Orthopedics 2015;38:684687.

8. Epps H. 3-D printing helps with complex hip surgery: Pediatric orthopaedists find it helpful for patient education too. AAOS Now 2014.

9. Hosny A, Shen T, Kuo AS, et al. Unlocking vendor-specific tags: Three-dimensional printing of echocardiographic data sets. J Thorac Cardiovasc Surg 2018;155:143-145.e1.

10. Chae MP, Rozen WM, McMenamin PG, et al. Emerging applications of bedside 3D printing in plastic surgery. Front Surg 2015;2:25.

11. Esses SJ, Berman P, Bloom AI, Sosna J. Clinical applications of physical 3D models derived from MDCT data and created by rapid prototyping. AJR Am J Roentgenol 2011; 196:W683-W688.

12. Matsumoto JS, Morris JM, Foley TA, et al. Threedimensional physical modeling: Applications and experience at mayo clinic. Radiographics 2015;35:1989-2006.

13. Bellanova L, Paul L, Docquier P-L. Surgical guides (patientspecific instruments) for pediatric tibial bone sarcoma resection and allograft reconstruction. Sarcoma 2013;2013:787653.

14. D'Urso PS, Earwaker WJ, Barker TM, et al. Custom cranioplasty using stereolithography and acrylic. Br J Plast Surg 2000;53:200-204.

15. Zopf DA, Hollister SJ, Nelson ME, Ohye RG, Green GE. Bioresorbable airway splint created with a three-dimensional printer. N Engl J Med 2013;368:2043-2045.

16. Kiarashi N, Nolte AC, Sturgeon GM, et al. Development of realistic physical breast phantoms matched to virtual breast phantoms based on human subject data. Med Phys 2015; 42:4116-4126.

17. Walker J, Leveille SG, Ngo L, et al. Inviting patients to read their doctors' notes: Patients and doctors look ahead: Patient and physician surveys. Ann Intern Med 2011;155:811-819.

18. Ripley B, Levin $\mathrm{D}$, Kelil $\mathrm{T}$, et al. 3D printing from MRI Data: Harnessing strengths and minimizing weaknesses. J Magn Reson Imaging 2017;45:635-645.

19. Bader C, Kolb D, Weaver JC, Oxman N. Data-driven material modeling with functional advection for 3D printing of materially heterogeneous objects. 3D Print Addit Manufactur 2016;3:71-79.

20. Brunton A, Arikan CA, Urban P. Pushing the limits of 3D color printing: Error diffusion with translucent materials. ACM Trans Graph 2015;35:1-13.

21. Reiner T, Carr N, Měch R, et al. Dual-color mixing for fused deposition modeling printers. Comput Graph Forum 2014;33:479-486.

22. Doubrovski EL, Tsai EY, Dikovsky D, et al. Voxel-based fabrication through material property mapping: A design method for bitmap printing. Comput Aided Des Appl 2015; 60:3-13. 
23. Fedorov A, Beichel R, Kalpathy-Cramer J, et al. 3D Slicer as an image computing platform for the Quantitative Imaging Network. Magn Reson Imaging 2012;30:1323-1341.

24. Floyd RW. An adaptive algorithm for spatial gray-scale. Proc Soc Inf Disp 1976; 17:75-77.

25. Bartlett NW, Tolley MT, Overvelde JTB, et al. A 3Dprinted, functionally graded soft robot powered by combustion. Science 2015;349:161-165.

26. Pobloth A-M, Checa S, Razi H, et al. Mechanobiologically optimized 3D titanium-mesh scaffolds enhance bone regeneration in critical segmental defects in sheep. Sci Transl Med 2018;10:eaam8828.

27. Patrick, GWSM. Massachusetts Institute of Technology. Growing a second skin: Towards synthetic biology in product design. Cambridge, MA: Massachusetts Institute of Technology, 2015.
28. Keating S. Beyond 3D printing: The new dimensions of additive fabrication. In: Designing for Emerging Technologies. Sebastopol, CA: O'Reilly Media, 2014, pp. 379-405.

29. Bader C, Kolb D, Oxman N, Weaver J. Methods and Apparatus for 3D Printing of Point Cloud Data. US Patent, 2017.

30. Comaniciu D, Engel K, Georgescu B, Mansi T. Shaping the future through innovations: From medical imaging to precision medicine. Med Image Anal 2016;33:19-26.

Address correspondence to: James $C$. Weaver Wyss Institute for Biologically Inspired Engineering Harvard University Cambridge, MA 02138

E-mail: james.weaver@wyss.harvard.edu 